\title{
Sustainability economics, resource efficiency, and the Green New
} Deal

\section{Journal Article}

\section{Author(s):}

Bretschger, Lucas

Publication date:

2010-08

Permanent link:

https://doi.org/10.3929/ethz-b-000029879

Rights / license:

In Copyright - Non-Commercial Use Permitted

\section{Originally published in:}

International Economics and Economic Policy 7(2-3), https://doi.org/10.1007/s10368-010-0168-6 


\title{
Sustainability economics, resource efficiency, and the Green New Deal
}

\author{
Lucas Bretschger, ETH Zurich
}

Published online: 26 June 2010

(C) Springer-Verlag 2010

\begin{abstract}
The paper addresses major sustainability issues within a simple general framework. It studies energy scarcity and endogenous capital formation in the long and medium run. It is shown that energy efficiency depends on the sectoral structure of the economy. Accordingly, structural change is an efficient way to promote both efficiency and sustainable development. The results for the medium run imply that the current crises offer a scope for the greening of the economy, provided that policy increases productivity through trust-building. However, there are major differences between economic recovery and sustainability so that the proposals of the Green New Deal have to be evaluated with care.
\end{abstract}

Keywords Sustainability • Resource efficiency • Trust • Green New Deal

JEL Classification $\mathrm{Q} 43 \cdot \mathrm{O} 47 \cdot \mathrm{Q} 56 \cdot \mathrm{O} 41$

\section{Introduction}

The sustainability debate suggests to aim at a long-run development which is characterized by non-decreasing living standards, a protection of crucial natural resources, and low risks of economic and ecological crises. Economic theory can provide basic insights on how such a sustainable path can be reached. It can also evaluate the usefulness of concrete proposals for a

L. Bretschger $(\bowtie)$

CER-ETH Centre of Economic Research, ETH Zurich,

ZUE F7, CH-8092 Zurich, Switzerland

e-mail: lbretschger@ethz.ch 
sustainable state, like the targets of $2 \mathrm{~kW}$ energy use or 1 ton $\mathrm{CO}_{2}$ emissions per capita.

Currently, climate change is the most imminent threat to sustainability. The business-as-usual scenario assumes that under laisser faire greenhouse gas emissions would rise by $45 \%$ by 2030 , which would cause an increase in the global average temperature of up to $6^{\circ} \mathrm{C}$. According to the Stern Review (Stern 2007), the warming could entail losses equivalent to 5-10\% of global GDP. Poor countries would suffer most with more than 10\% losses of GDP. Natural resource depletion and the loss of biodiversity are other critical issues for long-run sustainability.

Natural resources affect also the shorter-run development. Recently, we have experienced a triple crisis in the fields of food, fuel, and finance. Prices for food traded internationally increased by $60 \%$ in the first half of 2008 , oil price peaked at $150 \$ / \mathrm{barrel}$, and banking failures caused huge government interventions. Trade and per capita income have contracted worldwide in 2009 which implies one of the major economic downturns of the last decades.

The combination of these short-run developments with the long-term predictions lead to a variety of highly demanding research questions. Which mechanisms and activities are crucial to obtain sustainability? How can we decrease the long-term exposition to economic and environmental risks? Can expansionary governmental policy achieve two goals at the same time: stimulate recovery and improve sustainability?

The transition to a sustainable state implies a decarbonization of the economy and lower natural resource use. If welfare is to be sustained or increased in the future, the accumulation of man-made inputs consisting of different forms of capital has to be strong enough. The larger the saving effort of the present generation is, the better it is possible to substitute natural resources in production and consumption. The greatest challenge consists in showing that history of economic development can be reversed in the future: while in the past, low prices for the environment have led to extensive natural resource use and rising polluting activities, increasing prices of natural resource use should be able to change this general pattern. Corresponding to the concept of the Environmental Kuznets Curve (see e.g. Egli and Steger 2007), income should rise in the future while natural resource use should decrease.

Regarding the recent nexus between the crises and green policies there has been a widespread call for a "New Deal" as in the 1930s but at a global scale and embracing a broader vision, see Barbier (2009). The plan involves a sharp reduction in carbon intensity in order to revitalize the world economy on a more sustainable basis. Thus it is the aim to apply the same kind of long-run instruments for policies of a shorter time horizon.

The paper addresses these issues within a simple general framework. It applies the modelling of "new" resource economics, which includes a full characterization of the inherent dynamics and a sectoral structure of the economy. The second part concentrates on the medium run and its connection with sustainability issues, which are normally regarded as purely long term. It is shown that, in the long run, energy efficiency is a crucial issue for sustainability. 
However, we also derive that efficiency is not a pure technology parameter but depends heavily on the sectoral structure of the economy, which at the same time drives long-run growth. The results for the medium run imply that only a part of the proposals for the Green New Deal are promising because there are major differences between the aims of recovery and sustainability. In particular, government investments do not necessarily have the desired effects in the medium run.

The present contribution relates to the basic literature on resource economics and growth theory, in particular to Solow (1974a, b), Stiglitz (1974) and Dasgupta and Heal (1974). It relies on recent contributions of resource economics which have widened our knowledge on sustainability, see Bovenberg and Smulders (1995), Barbier (1999), Bretschger (1998, 1999), Scholz and Ziemes (1999), Smulders (2000), Grimaud and Rougé (2003), Xepapadeas (2006), and López et al. (2007), and Bretschger and Smulders (2008).

The remainder of the paper is organized as follows. Section 2 presents an approach to obtain sustainability results with a focus on resource efficiency. In Section 3, specific results for the long run are derived. Section 4 introduces the elements of medium-run analysis and the Green New Deal. Section 5 shows results for the comparative dynamics of the model. Section 6 concludes.

\section{Efficiency focus}

In the following, we develop a general framework to study major sustainability issues. The model has some specific features depending on the considered time horizon. For the long run, we allow for all possible substitution mechanisms between inputs and sectors which characterize the long-term flexibility of a market economy. For the medium run, limited substitution between inputs and the emergence of business cycles will be the focus of the study.

We start by analyzing aggregate production and the implications for energy efficiency. Instead of adopting a one-sector approach with capital, labor, and energy as inputs we assume that production is characterized by a hierarchical order as follows: final output is manufactured by "produced" inputs and produced inputs are manufactured by primary inputs. This enables us to express the different substitution channels in a simple yet comprehensive manner. The distinctive feature of the model is that the impact of energy on output occurs indirectly, through the produced inputs. It does not mean that energy is unimportant for final goods. On the contrary, energy affects all production processes, including final goods, but in a more detailed way compared to the one-sector model.

Assume output $Y$ as a function of total factor productivity $A$ and the produced inputs capital $K$ and intermediate input $Z$ :

$$
Y(t)=F[A(t), K(t), Z(t)]
$$


with a convenient specification reading:

$$
Y(t)=A(t) K(t)^{\alpha} Z(t)^{1-\alpha}
$$

where $t$ denotes the time index and $0<\alpha<1$. Produced inputs $K$ and $Z$ are manufactured in separate sectors of the economy with the primary inputs labor $L$ and energy $E$, according to:

$$
\begin{aligned}
& \dot{K}(t)=L_{K}(t)^{\beta} E_{K}(t)^{1-\beta}-\delta K(t) \\
& Z(t)=L_{Z}(t)^{\gamma} E_{Z}(t)^{1-\gamma}
\end{aligned}
$$

where $\dot{K} \equiv \partial K / \partial t, \delta$ is the depreciation rate, $0<\beta, \gamma, \delta<1$, and the subscripts with inputs denote the sectors. $E$ includes all types of energies and natural resources. Capital $K$ is a broad measure of accumulable inputs. It can be thought of as an aggregate of physical, human, and (private) knowledge capital. Positive spillovers from capital accumulation raise $A$ (as introduced by Arrow 1962), the general productivity of inputs; $A$ can also be interpreted as public knowledge. Material balances define the limits to physical capital, but not to the other capital types. Total energy efficiency $x$ affects output according to:

$$
Y \equiv \frac{F(\cdot)}{E} E=x \cdot E
$$

where $F$ is determined by (1). Importantly, $x$ is not a single technology parameter as often referred to in applied studies, but rather depends on all the model parameters including production in every sector of the economy. In the steady state ( $\dot{K}=0 ; \alpha+\eta<1$ ) it reads, using (2), (3), and (4):

$$
x=\frac{A(t) \delta^{-\alpha} L_{K}(t)^{\alpha \beta} L_{Z}(t)^{(1-\alpha) \gamma} E_{K}(t)^{\alpha(1-\beta)} E_{Z}(t)^{(1-\alpha)(1-\gamma)}}{E(t)}
$$

It becomes clear from (6), that a change of $x$ is not necessarily due to a change of total factor productivity $A$. To increase $x$ with a given $A$, there are options such as a decrease of $\delta$, an increase of $L$ (with $L=L_{K}+L_{Z}$ ), or a reallocation of primary inputs $L$ and $E$ between the different sectors. Thus, energy efficiency is as much an economic and societal result as it is an engineering issue. The effect of a reallocation of primary inputs between the sectors depends on the technology parameters $\alpha, \beta, \gamma$. For example, when reallocating labor from $Z$ - to $K$-production, following (6) the impact depends on whether $\alpha \beta$ is bigger or smaller than $(1-\alpha) \gamma$, which are the exponents of $L_{K}(t)$ and $L_{Z}(t)$.

Three remarks concerning the demand side of the economy are in order. The first concerns the level of the steady state capital stock used to calculate (6), which depends on the savings behavior of the households. A higher savings rate enables the economy to accumulate more capital which affects energy efficiency positively as well. Second, an increasing $L$ raises ceteris paribus energy demand of consumers which has to be considered as well. The third remark is about the impact of trade, which affects the domestic economy 
by the terms of trade. Specifically, a very productive (e.g. energy efficient) economy produces a high output which is confronted with demand conditions on world markets. In general, higher efficiency increases output which worsens the terms of trade, except the domestic economy is very small. This means that efficiency affects domestic consumption also through the terms of trade effect.

In the following, we analyze long-run and medium-run development in greater detail. Specifically, the long-run sustainability view is first developed and then related to the issue of economic recovery as currently discussed in many countries. Here, the relationship between cycles and growth is the dominant topic to determine efficiency. This connects the model to the debate on the Green New Deal.

\section{Long-run analysis}

Let us now turn to the long-run dynamic aspects of energy efficiency in the present framework. We study the effects of decreasing energy input, which may be the consequence of climate policies or limited resource supply. With hats denoting growth rates and assuming the change of energy input to be negative it follows from (5) that for output to grow:

$$
\hat{x} \geq-\hat{E} \quad \text { for } Y \geq 0
$$

has to hold. Equation 7 says that we can directly calculate the efficiency increase $\hat{x}$ needed for constant or increasing output, as soon as we know the change of energy input $-\hat{E}$. Evidently, for a growing output, efficiency $x$ has to rise faster than energy use $E$ decreases. To see the implications, we have to study the long-run impact of energy $E$ on output. Logarithmic differentiating (2) yields:

$$
\hat{Y}(t)=\hat{A}(t)+\alpha \hat{K}(t)+(1-\alpha) \hat{Z}(t)
$$

It is important to note that, with limited supply of $L$ and $E, Z$ is bounded from above, which follows from (4). ${ }^{1}$ That is, we have $\hat{Z}(t)=0$ in the long run. However, $K$ is substantially different from $Z$ as it is a stock, which accumulates over time. Even with a bounded quantity of primary inputs we can produce an increasing stock of capital. Moreover, it is important to note that $A$ and $K$ are interlinked by positive spillovers, according to Arrow (1962). This means that capital accumulation has a positive impact on factor productivity through learning by doing. In order to avoid the scale effect of growth, see Jones (1995) and Peretto (2009), we can assume that spillovers are generated through an increase of the average capital stock in order to write:

$$
A(t)=\tilde{A} \frac{K(t)^{\eta}}{Z(t)^{1-\alpha}}
$$

\footnotetext{
${ }^{1} E$ is the per-period flow of energy, which is generally limited for non-renewable resources and also limited for renewables, provided that they cannot be infinitely expanded, which we assume.
} 
where $\tilde{A}>0$ is a parameter, $0<\eta<1$ denotes the spillover intensity, and we divide by $Z^{1-\alpha}$ to eliminate the scale of growth. ${ }^{2}$ Now, (2) becomes:

$$
Y(t)=\tilde{A} K(t)^{\alpha+\eta}
$$

According to (10), the only variables which drive development in the long run are $K, \alpha$ and $\eta$. A convenient choice is $\alpha+\eta=1$ so that we arrive at the class of the so-called "AK-models" of endogenous or linear growth. Here, capital growth directly translates into output growth, i.e. $\hat{Y}=\hat{K}$. From (3) we have:

$$
\hat{K}=\frac{L_{K}(t)^{\beta} E_{K}(t)^{1-\beta}}{K(t)}-\delta
$$

Equation 11 exhibits that it is not total energy $E$ that has a direct impact on growth. Rather the sectoral input of energy in capital accumulation $E_{K}$, together with sectoral labor $L_{K}$, matters for the growth rate. The lower the energy input $E_{K}$ is, the more important becomes labor used in capital production $L_{K}$ to sustain the growth process.

Equation 11 says that, to determine the impact of $\hat{E}$ on $\hat{Y}$, we have to study the effect of $\hat{E}$ on $L_{K}$ and $E_{K}$ which determine $\hat{K}$. There are two different mechanisms to consider. On the one hand, there is a demand effect, which exhibits how demand for capital affects input use in capital production. Observing the general production relation (1), $\hat{E}<0$ affects the demand for capital by $Y$-producers through a change in the output share of capital. Provided that the elasticity of substitution between $K$ and $Z$ is high (i.e. above unity), the income share of capital increases and the demand for capital rises which fosters growth. With the same argument, a low input elasticity between $K$ and $Z$ depresses the reward for capital which decreases growth prospects. This is the well-known result of resource economics in the 1970s, see Dasgupta and Heal (1974). With the Cobb Douglas specification (2), of course, these shares remain exactly constant and the effect does not materialize. In this case, a change of energy input has no impact on capital accumulation through the demand effect.

While for the demand effect we aim at a high substitution elasticity for sustainability, the same does not necessarily hold true for the supply effect which deals with the cost of capital accumulation. To see the cost effect observe that $E$ is an input both in (3) and (4). With the given Cobb Douglas specification it is again that shares remain constant and labor cost remain unaffected by energy prices and quantities. To see the supply effect, assume for a moment that the shares $\beta$ and $\gamma$ are not constant but depend on relative input prices. This means that the Cobb Douglas specifications in (3) and (4) are replaced by more general CES functions where the elasticity of substitution between $L$ and $E$ is unequal one. Then, $\hat{E}<0$ causes input substitution and

\footnotetext{
${ }^{2}$ The division by the intermediate input is convenient but not necessary for the argument.
} 
an induced change of output in both activities. Specifically, labor moves to the sector where wages become (relatively) more attractive which is the sector with the higher elasticity of substitution. Provided that substitution possibilities are better in capital accumulation than in the production of $Z$, increasing energy scarcity causes $L$ to move from the $Z$-sector to the capital sector which enhances capital accumulation and the growth of energy efficiency and output. Therefore, $\hat{x}$ does not depend on the absolute values of the elasticities of input substitution but on their relative values. Poor input substitution elasticities are not necessarily detrimental for growth. For sustainability, the elasticity of substitution has to be higher in the capital accumulation sector compared to the competing sector, which is the $Z$-sector, see Bretschger and Smulders (2008) for a more detailed analysis. Hence, sustainability depends not only on input substitution but also on sectoral change, which promotes growth, when primary inputs are reallocated towards capital accumulation.

The model can be extended to more types of capital. Specifically, $K$ can be disaggregated into physical, human, and (private) knowledge capital. For these different capital types, the described demand and supply effects can be studied separately. ${ }^{3}$ The interesting feature of this approach is that it has neither to assume high elasticities of input substitution or an ever increasing use of material input to predict sustainable development, which were the assumptions of earlier models criticized by ecologists, see Cleveland and Ruth (1997).

\section{Medium-run analysis}

For the analysis of the medium run, we introduce business cycles and consider the effects of zero input substitution. As an additional model element, we introduce a variable for the trust level in the economy. We then focus on cycles emerging from trust building through capital accumulation. Accordingly, the capital build-up is associated with social learning which entails trust and confidence. We assume that, besides the long-run positive learning effects of capital accumulation, there is also cyclical learning in the economy. Trust and confidence gradually build up during a cycle. They reflect broad areas such as trust in rules and institutions, the emergence of implicit contracts, and the efficiency of additional markets (like the interbank market). These issues affect people's willingness to cooperate and thus the productivities of the inputs.

We can model the emergence and disappearance of trust as an externality, like the positive spillovers. Specifically, we assume that the start of a cycle is triggered by an exogenous event, e.g. a new technology or a specific policy; examples are the appearance of the internet or the home-owning initiative of the US government. But then, after a certain time, trust building fades

\footnotetext{
${ }^{3}$ Empirical evidence suggests that a decrease in energy use fosters investments in physical and knowledge capital and is neutral with regard to human capital, see Bretschger (2009).
} 
Fig. 1 Trust and cycle

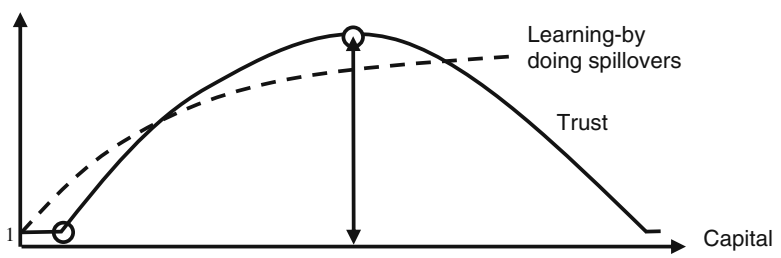

so that the additional productivity deteriorates. Thus while the economic upswing is achieved through an increasing trust in the new paradigm, the economic downturn is due to learning about problems and misjudgements of the paradigm. Possibly, new cycles emerge during or after this process. Figure 1 shows the relationship between capital and learning graphically, during one cycle.

In the following, $B_{j}$ denotes the size of trust in the $j$ th cycle; it is determined by positive spillovers from capital accumulation. We have $B_{j}=0$ until a technology or policy shock occurs (which happens at $K=K_{j}$ ). The development of $B_{j}$ is determined by the "trust dissemination rate" $\kappa$ and the "maximum obtainable" trust $\bar{B}_{j}$. $\kappa$ reflects communication within the business community and the political sector (e.g. electronic media are assumed to increase $\kappa$ ). $\bar{B}_{j}$ depends on the perceived potential of the new paradigm, i.e. the productivity enhancing effect of the new cycle. $\kappa$ and $\bar{B}_{j}$ together determine when the cycle ends (which happens at $K=\bar{K}_{j}$ ). For simplicity, we assume $B \geq 1$ below. ${ }^{4}$ Adopting a logistic form for trust building through capital accumulation we get:

$$
B_{j}(t)=\max \left\{\kappa \cdot K(t)\left[1-K(t) / \bar{K}_{j}(t)\right], 0\right\}+1
$$

so that

$$
\bar{K}_{j}(t)=\left(4 \bar{B}_{j}-1\right) / \kappa+\underline{K}_{j}(t)
$$

which determines the end point of cycle $j$ and its length, see the Appendix for the derivation.

In the medium run, energy and capital are assumed to be pure complements. However, energy-saving investments are now feasible. We define $\tilde{K}$ as:

$$
\tilde{K}(t)=\min \{B(t) \cdot K(t), D(t) \cdot E(t)\}
$$

where $B$ is given by (12) and $j$ is omitted from now on because there is no ambiguity. $D$ is a partial energy efficiency parameter (note that in general $D \neq x$ ). With given technology (fixed $D$ ), $E$ and $B(t) K(t)$ are pure complements. However, substitution between $D$ and $E$ is possible, the substitution elasticity is unity, as usual. $\tilde{K}$ increases with $K$ when $E$ and/or $D \operatorname{rise(s).~} D(t)$ can be increased by research investments.

\footnotetext{
${ }^{4}$ To take $B$ positive but smaller than unity would be feasible as well.
} 
Moreover, we assume for simplicity in the medium-run analysis that the production technologies of $Y$ and $K$ are symmetric and of the Cobb-Douglas form. In this way, (3) can be simplified and $K$ accumulates according to:

$$
\dot{K}(t)=s \cdot Y(t)-\delta K(t)
$$

where $s$ is the savings rate. $A(t)$ now reads:

$$
A(t)=\tilde{A} \frac{\ddot{K}(t)^{\eta}}{Z(t)^{1-\alpha}}
$$

where $\ddot{K}$ is actually used capital $(\ddot{K}<K$ when $B K<D E)$ and we again divide by $Z^{1-\alpha}$ to eliminate the scale effect. During a cycle, maximum output is obtained with $B(t) \cdot K(t)=D(t) \cdot E(t)$, which applies when energy supply is fully elastic. Then, the model predicts cyclical energy use.

Regarding the supply of energy we argue along two possible scenarios. In regime 1 ("affluent energy"), energy supply is fully elastic at given energy prices $\bar{p}_{E}$ :

$$
p_{E}=\bar{p}_{E}
$$

Growth in regime 1 is then determined by $B K=D E, \tilde{K}=B K$, and $\ddot{K}=K$ so that we obtain:

$$
\begin{aligned}
& Y=\tilde{A} B^{\alpha} K^{\alpha+\eta} \\
& \hat{K}=s \tilde{A} B^{\alpha} K^{\alpha+\eta-1}-\delta
\end{aligned}
$$

In regime 2 ("limiting energy"), energy supply is restricted according to:

$$
E=\bar{E}<B \cdot K / D
$$

Growth in regime 2 is thus bounded by energy supply $\bar{E}$.

Figure 2 exhibits the different possible developments paths for the economy. The geometrical loci labelled with $Y$ denote output with $Y=\tilde{A} B^{\alpha} K^{\alpha+\eta}$ in regime 1 and $Y$ depending on $E$ in regime 2. The case for regime 1 with $\alpha+\eta<1$ is depicted with the solid curved line. Long-term learning spillovers $(\eta>0)$ shift development upward, i.e. $Y=\tilde{A} B^{\alpha} K^{\alpha+\eta}>Y=\tilde{A}(B K)^{\alpha}$. The cyclical impact of trust is added by the dotted semicircles above the curve. If positive learning effects are strong we may have $\alpha+\eta=1$ and get constant returns to capital, which leads to the solid straight line for $Y_{s}{ }^{5}$ If energy is the limiting factor as in regime 2, output becomes lower (an example is given with the dashed line for $Y_{d}$ ), with fading energy input and constant $D$ it even approaches zero in the long run. When, in addition, temporary energy shocks in form of intensified shortages hit the economy (like in the 1970s), output

\footnotetext{
${ }^{5}$ Trust is not added in this case but used in the following figures.
} 
Fig. 2 Different development paths

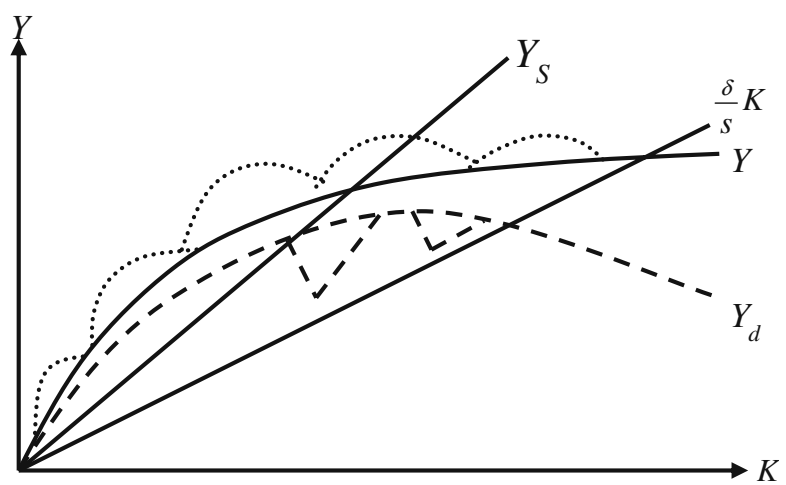

deviates downward (see the dotted downward deviations). As the steady state assuming $\alpha+\eta<1$ is defined by $\dot{K}=0$, the intersection of the $Y$-loci with the $\delta K / s$-locus yields the long-run equilibrium.

In the following, we use these scenarios for the discussion of the different policies.

\section{Comparative dynamics}

In this section, we present several policies associated to the Green New Deal and their effects on output according to the assumptions of the mediumrun model. The results for the two energy regimes are presented. It is most instructive to present the results graphically with a short verbal description; the summary discussion is given in the last subsection.

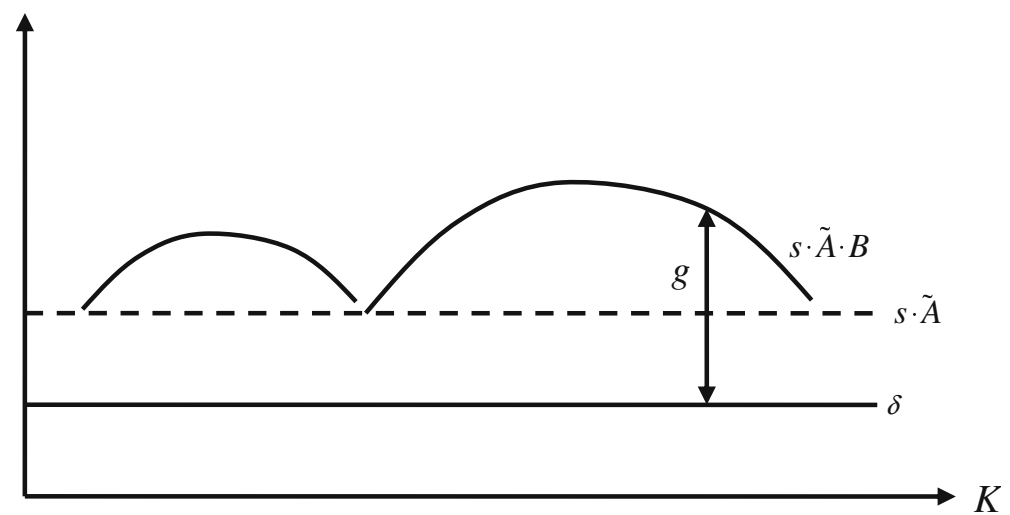

Fig. 3 Growth in regime 1 

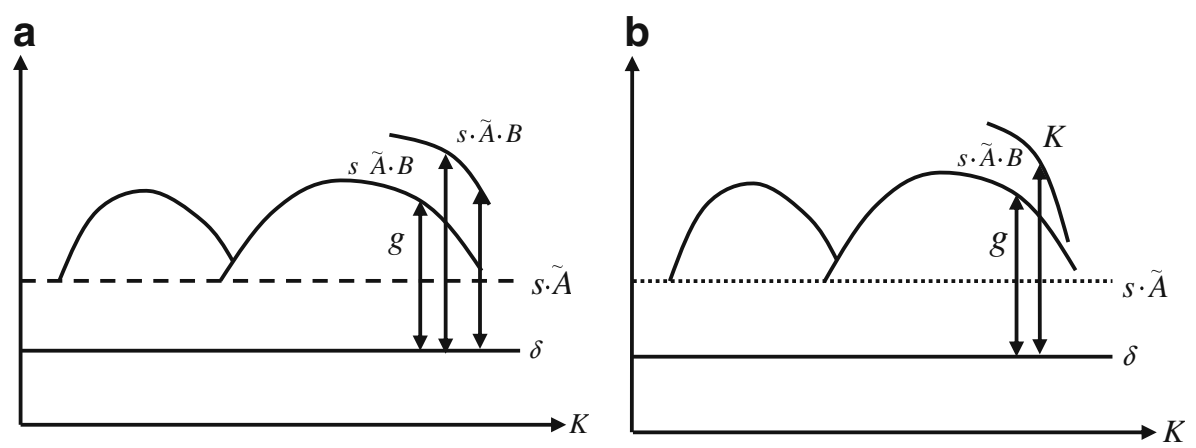

Fig. 4 Impact of subsidies and trust building

\subsection{Regime 1 (affluent energy)}

When energy supply is fully elastic, we have $B K=D E$ which entails $\tilde{K}=B K$ and $\ddot{K}=K$. Assuming that $\alpha+\eta=1$ we get for capital growth:

$$
\hat{K} \equiv g=s \tilde{A} B^{\alpha}-\delta
$$

Figure 3 shows the cyclical growth rate of capital graphically.

Possible policies in the spirit of the Green New Deal are to subsidize savings $s$, to affect trust building $\kappa$, to affect depreciation $\delta$ or to launch a new cycle $B$. Let us consider the effects in turn. We first show graphical representations of the results for the two energy regimes and then discuss the impact of policy in a summary at the end of the section.

When the government decides to subsidize savings $s$, the direct effect on growth is positive but overall growth might still decrease because of shrinking $B$, see Fig. 4a. When the government decides to support trust building in the present paradigm (by e.g. reinforcing a paradigm-specific policy) it increases growth in the short run but cannot prevent growth from decreasing at the end of the cycle, see Fig. $4 b$.

Provided that investments are reallocated to more sustainable sectors or processes (minergy housing, hybrid cars) it is conceivable to assume that depreciation increases in the medium run which harms growth, see Fig. 5a. ${ }^{6}$ Only in the case in which the government is able to start a new trust cycle (a new paradigm), growth increases in the short and medium term, see Fig. 5 b.

\footnotetext{
${ }^{6}$ In parallel, employment might get hit because the reallocation of workers can cause adjustment costs, but this is not included in the model.
} 

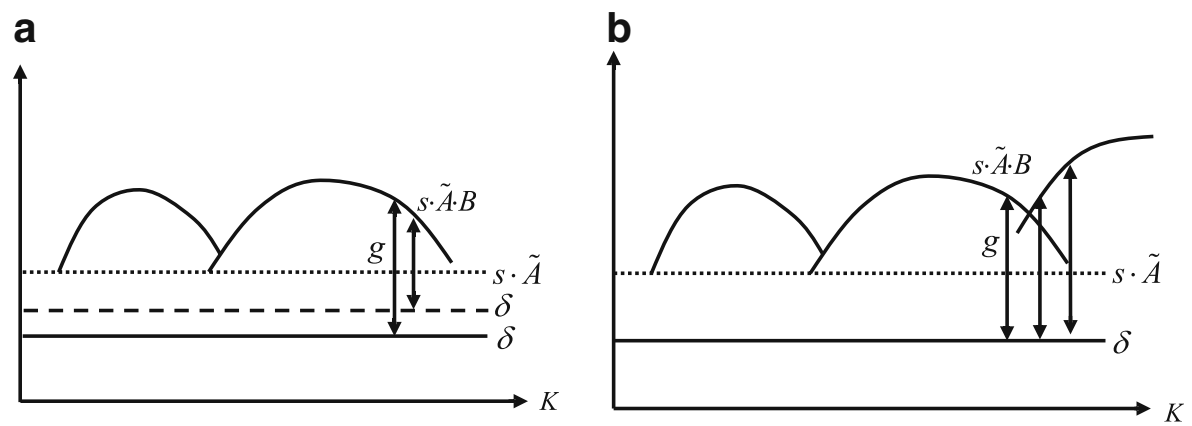

Fig. 5 Impact of depreciation and new trust cycle

\subsection{Regime 2 (limiting energy)}

Assuming that $B K>D E$ we get $\tilde{K}=D E$ and $\ddot{K}=D E / B<K$ so that:

$$
\begin{aligned}
& Y=\tilde{A} \cdot B^{-\eta} \cdot(D E)^{\alpha+\eta} \\
& \hat{Y}=-\eta \hat{B}+(\alpha+\eta)(\hat{D}+\hat{E})
\end{aligned}
$$

Increasing trust $B$ now involves a countercyclical effect, i.e. it has a negative impact on income and growth (because $A$ depends on $\ddot{K}$, not on $K$, see (16)). Capital increases according to $\hat{K}=s \cdot \tilde{A} B^{-\eta}(D E)^{\alpha+\eta}-\delta$ but only investments in energy-savings are relevant for output according to:

$$
\begin{aligned}
\dot{D} & =s_{D} Y \\
& =s_{D} \cdot \tilde{A} B^{-\eta}(D E)^{\alpha+\eta}
\end{aligned}
$$

Assuming $\alpha+\eta=1$, output growth is given by:

$$
\hat{Y}=-\eta \hat{B}+\hat{D}+\hat{E}
$$

which is represented in Fig. 6, still assuming $\hat{E}<0$.

Fig. 6 Growth in regime 2

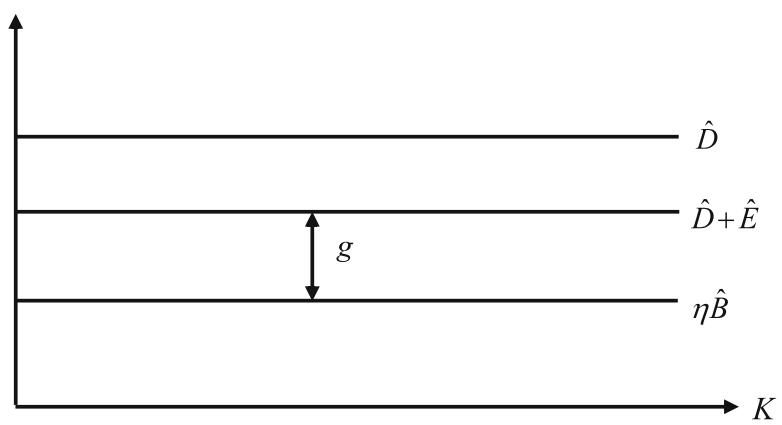



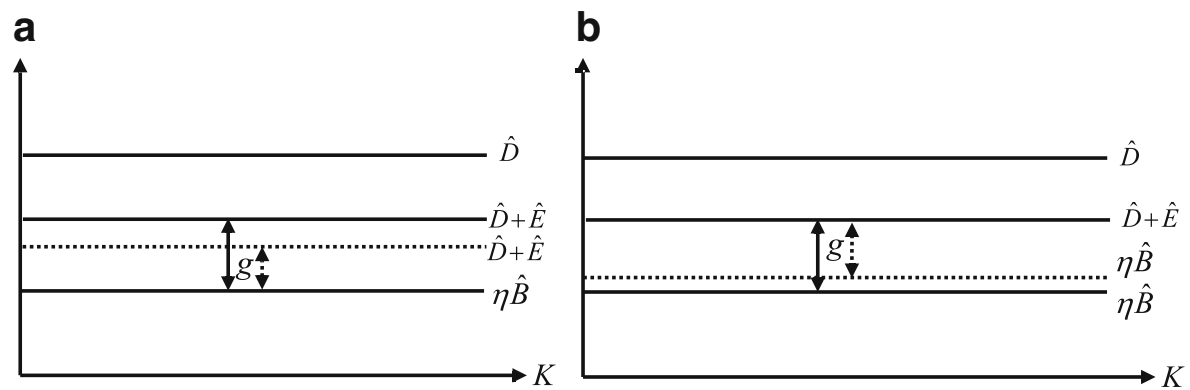

Fig. 7 Impact of energy and savings

Possible policies in the wave of the Green New Deal concern instruments to change energy use, to subsidize savings $s$, and to subsidize energy-saving technology through raising $s_{D}$. To decrease energy use has a negative effect on growth in the medium run, see Fig. 7a. ${ }^{7}$ Remarkably, rising capital investments by increasing $s$ has a negative effect on growth in this case, because capital becomes more productive through an increase in trust $(\hat{B}>0)$, so that less capital is used and spillovers are reduced, see (23) and Fig. 7b.

The best policy concerns an improvement of energy saving technologies, because it leads to a relaxation of the energy supply constraint and unambiguously raises growth, see Fig. 8.

In regime 2, energy $E$ affects output $Y$ according to

$$
Y(t)=\ddot{K}(t)^{\eta}[D(t) \cdot E(t)]^{\alpha} L(t)^{1-\alpha}
$$

where $\ddot{K}$ denotes capital in use $(\ddot{K}=D E / B<K)$. Thus with given $\tilde{K}, D$, and $L$, output $Y$ directly depends on $E$. Moreover, decreasing energy input limits long-run development prospects (see Fig. 2), provided that improvements in energy efficiency $B$ cannot compensate for fading energies.

\subsection{Policy assessment}

From the above results of comparative dynamics we derive that, in a mediumrun downturn, increasing the savings rate $s$ and associated capital investments through governmental policy shortens the downturn time of the cycle but does not necessarily increase (medium-run) income because trust $B$ is decreasing. This holds true unless policy is able to increase new trust, i.e. to start a new paradigm, where income increases with a new cycle. We have thus to ask how likely it is that a new paradigm can arise through the implementation of green policies.

\footnotetext{
${ }^{7}$ This result is not necessarily identical to the long run, see Section 3.
} 
Fig. 8 Impact of higher energy efficency

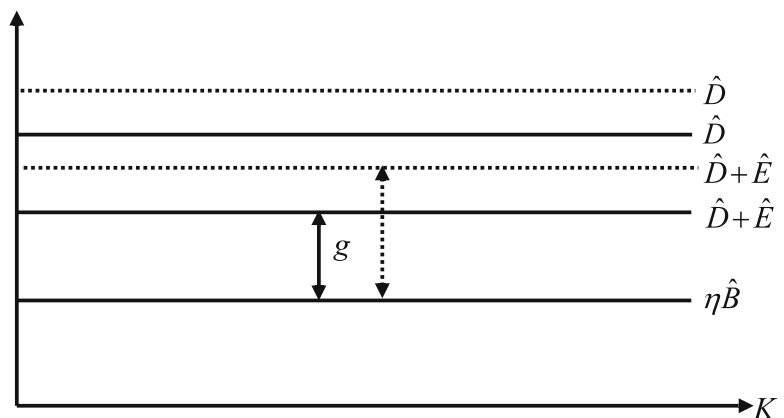

Increasing capital investments by governmental policy possibly involves two further problems. First, it has no impact on long-run income, provided that energy is scarce (regime 2). Second, if we are in regime 1, increasing capital investments raise energy demand thereby raising exposure to risk of future energy supply shortages.

Increasing energy efficiency by governmental policy supports the development of long-run income in regime 2. At the same time, it decreases long-run energy demand and reduces the risk in the case of energy shortages as with low energy input, the term $B(t) \cdot E(t)$ is dominated by efficiency $B(t)$. When energy is abundant (regime 1 ), increasing energy efficiency by governmental policy reduces energy use but does not foster income and growth.

\section{Conclusions}

The model used in this paper provides results for sustainability policies in the long and medium run. For the long run, substitution between inputs and, above all, between sectors is necessary to move the economy towards higher resource efficiency and to enable ongoing growth. Medium-run policies may aim at escaping an economic downturn with several measures summarized under the title "Green New Deal". We conclude that, in principle, it is a valid point to direct government expenditure toward a greening of the economy, if these expenditures are carried out anyway. But it has to be noted that mediumrun recovery is not the primary target of sustainability policies. For example, with regard to future living standards and risk exposure, sustainability calls for policies increasing energy efficiency rather than raising capital investments. Assuming that sustainability policies create new trust brings the goals of recovery and sustainability in line, as claimed by the Green New Deal. But is it realistic? Further problems of large green programmes with huge government spending are the lack of mature energy projects (causing low efficiency) and possibly high administrative costs. In addition, these policies might carry a green label but in fact only help existing industries to survive (like the German "scrap premium"). Finally, taxes and permit markets can have similar or better effects, without causing a high burden for public budgets. 
Except the explanations on the terms of trade effect we did not treat the international dimension in this paper. Of course, the state of the environment and economic growth are both largely influenced by the economic relations between economies and world regions. Thus the combination of dynamics, trade and environment is a promising field for further economic research. Looking at the existing literature indicates that more interesting results can be expected in the future. Many results of growth theory are only valid for closed economies; by opening the economies, one should try to confirm, reject or refine these model outcomes.

\section{Appendix}

\section{Calculation of B}

$$
\begin{aligned}
B_{j}(t)= & \kappa \cdot K_{j}(t)-\kappa \cdot K_{j}(t)^{2} / \bar{K}_{j}(t)+1 \\
\bar{B}_{j} & : \frac{\partial B_{j}(t)}{\partial K_{j}(t)}=0 \\
& \Leftrightarrow \kappa=2 \cdot \kappa \cdot K_{j}(t) / \bar{K}_{j}(t) \\
& \Leftrightarrow \bar{K}_{j}(t)=2 \cdot K_{j}(t) \\
& \Leftrightarrow K_{j}(t)=\bar{K}_{j}(t) / 2
\end{aligned}
$$

Inserting in the logistic function gives

$$
\begin{aligned}
\bar{B}_{j}(t) & =\kappa \cdot \bar{K}_{j}(t) / 2\left[1-\bar{K}_{j}(t) / 2 \bar{K}_{j}(t)\right]+1 \\
& =\kappa \cdot \bar{K}_{j}(t) / 2 \cdot 1 / 2+1 \\
& =\kappa \cdot \bar{K}_{j}(t) / 4+1
\end{aligned}
$$

so that $\bar{K}_{j}(t)=4\left(\bar{B}_{j}-1\right) / \kappa$ if $\underline{K}_{j}(t)=0$ and $\bar{K}_{j}(t)-\underline{K}_{j}(t)=4\left(\bar{B}_{j}-1\right) / \kappa$ if $\underline{K}_{j}(t)>0$ as used in the main text.

\section{References}

Arrow KJ (1962) The economic implications of learning by doing. Rev Econ Stud 29:155-173

Barbier EB (1999) Endogenous growth and natural resource scarcity. Environ Resour Econ 14(1):51-74

Barbier EB (2009) A global Green New Deal. Report prepared for the Green Economy Initiative and the Division of Technology, Industry and Economics of the UN Environment Programme

Bovenberg AL, Smulders S (1995) Environmental quality and pollution-augmenting technological change in a two-sector endogenous growth model. J Public Econ 57:369-391

Bretschger L (1998) How to substitute in order to sustain: knowledge driven growth under environmental restrictions. Environ Dev Econ 3:425-442

Bretschger L (1999) Growth theory and sustainable development. Edward Elgar, Cheltenham UK Bretschger L (2009) Energy prices, growth, and the channels in between: theory and evidence. CER-ETH Economics Working Paper Series 06/47, ETH Zurich 
Bretschger L, Smulders S (2008) Sustainability and substitution of exhaustible natural resources; how resource prices affect long-Term R\&D-Investments. CER-ETH Economics Working Paper Series 03/26, ETH Zurich

Cleveland C, Ruth M (1997) When, where and by how much do biophysical limits constrain the economic process; a survey of Nicolas Georgescu-Roegen's contribution to ecological economics. Ecol Econ 22:203-223

Dasgupta PS, Heal GM (1974) The optimal depletion of exhaustible resources. Rev Econ Stud 41:3-28

Egli H, Steger T (2007) A dynamic model of the environmental Kuznets curve: turning point and public policy. Environ Resour Econ 36(1):15-34

Grimaud A, Rougé L (2003) Non-renewable resources and growth with vertical innovations: optimum, equilibrium and economic policies. J Environ Econ Manage 45:433-453

Jones C (1995) R\&D-based models of economic growth. J Polit Econ 103:759-784

López R, Anriquez G, Gulati S (2007) Structural change and sustainable development. J Environ Econ Manage 53:307-322

Peretto P (2009) Energy taxes and endogenous technological change. J Environ Econ Manage 57/3:269-283

Scholz CM, Ziemes G (1999) Exhaustible resources, monopolistic competition, and endogenous growth. Environ Resour Econ 13:169-185

Smulders S (2000) Economic growth and environmental quality. In: Folmer H, Gabel L (eds) Principles of environmental economics. Edward Elgar, Cheltenham UK, chapter 20

Solow RM (1974a) Intergenerational equity and exhaustible resources. Rev Econ Stud 41:29-45

Solow RM (1974b) The economics of resources or the resources of economics. Am Econ Rev 64:1-14

Stern N (2007) The economics of climate change. Cambridge University Press, Cambridge

Stiglitz JE (1974) Growth with exhaustible natural resources: efficient and optimal growth paths. Rev Econ Stud 41:123-137

Xepapadeas A (2006) Economic growth and the environment. In: Mäler K-G, Vincent J (eds) Handbook of environmental economics. Elsevier Science, Amsterdam 